Research Article

\title{
Thermoelastic Characteristics of Functionally Graded Circular Disk Models under the Loading of Contact Forces
}

\author{
Jaegwi Go \\ Department of Mathematics, Changwon National University, 20 Changwondaehak-ro Uichang-gu, Changwon-si 51140, \\ Gyeongsangnam-do, Republic of Korea \\ Correspondence should be addressed to Jaegwi Go; jggo@changwon.ac.kr
}

Received 29 September 2020; Revised 30 November 2020; Accepted 22 December 2020; Published 6 January 2021

Academic Editor: Fateh Mebarek-Oudina

Copyright () 2021 Jaegwi Go. This is an open access article distributed under the Creative Commons Attribution License, which permits unrestricted use, distribution, and reproduction in any medium, provided the original work is properly cited.

A rotating functionally graded circular disk undergoing a contact load is taken into account to investigate the thermoelastic characteristics. By considering contact force, a pair of partial differential equations is induced as the governing equations based on Hooke's law. The behavior of circular disk modes is described with the variations of contact force and homogeneous thickness. A finite volume model is introduced to obtain approximate solutions for the governing equations because of the complexity of the equations. Contact force is highly influential in the radial direction compared to the circumferential direction in the displacement distribution, while a large radial stress appears near the area of the contact point. In the strain distribution, the magnitude increases as the angle grows near part of the outer boundary in the circular domain. The radial distribution profiles are susceptible to the growth of contact force in nearby area of the outer boundary, whereas the influences on the circumferential direction profiles are trivial. The increase of homogeneous thickness dwindles the radial magnitude of displacement, stress, and strain distribution profiles over nearby area of the outer boundary of the circular domain. As a result, numerical approach demonstrates that contact force and homogeneous thickness are indispensable parameters and provide deep influence on the thermoelastic movements of a rotating circular disk. Thus, the results obtained may be useful to design an appropriate FGM circular disk model for the industrial area by controlling the above parameters.

\section{Introduction}

The analysis of thermoelastic characteristics of a rotating circular disk has been a challenging work due to complex processes generating during operations. Friction, wear, heat generation, and temperature deformation are representative processes developed during the load of a contact force. By the operation of a rotating circular disk, all parameters are connected mutually and complicated interactions progress. The thermoelastic characteristics of a circular disk are under complicated interaction influences and the variation of each parameter may not be ignorable to determine the thermoelastic movements. Thus, the influences of temperature, contact pressure, and blushed wear on the rotating circular disk are interesting and valuable for research.

Ceramic is a typical material used at one surface to resist severe environmental effects such as high temperature, wear, and friction. The other surface is composed of a metal to ensure higher toughness and thermal conductivity. The complementary functions of each material invoke the introduction of functionally graded materials (FGMs). FGMs are nonhomogeneous composites composed of two or more different material phases and the volume fractions continuously vary with space variables. Thus, FGMs are structures involving successively varying mechanical and thermal properties from one surface to another surface. FGMs were introduced to prosper thermal barrier coatings for propulsion system of space planes to endure high temperature and ensure high thermal conductivity at the beginning [1]. The graded material property provides improved bonding strength in various applications undergoing a high temperature gradient field.

On the pursuit superior properties such as strength, impact toughness, and fiber resistance, fiber metal laminates (FMLs) have drawn attention as promising hybrid 
composite materials in last few decades. FMLs are composed of metallic and composite materials. The combination of fiber-reinforced composite layers and composite materials creates FMLs, which exhibit excellent durability. Thus, FMLs are applicable in various industries and are used in aircraft, wind turbine, and automobile. A laminated composite made with thin sheet/grass was investigated by BnvsGaneshGupta et al. [2] for the flexural properties. They evaluated flexural strength and failure analysis using stereo microscope. Zimmermann and Wang [3] analyzed failure modes and characteristics of adhesively and mechanically fastened joints in aircraft composite materials.

Meanwhile, the shape of circular disk is commonly employed for practical use in various industrial applications, and the physical behavior of circular disk models is a matter of interest to many engineering researchers. Obata and Noda [4] and Liew et al. [5] analyzed the thermal stresses of FGM circular cylinder and hollow sphere. Afsar et al. [6] investigated the brittle fracture characteristics of thick-walled FGM cylinders by considering incompatible eigenstrain, which is a cause of thermal residual stress. The characterization that oscillatory movements reduce cutting forces in operating process of a circular disk cutter was presented by Kovalyshen [7], and Huang and $\mathrm{Li}$ [8] studied the radial component effects on the bending of thin circular plates using Kirchhoff plate theory. The angular speed of a rotating circular disk varies during the start and stop process in machine operation. Dai et al. [9] investigated the displacement and stress fields of a FGM hollow circular disk subjecting an angular acceleration due to a changing temperature. Static behavior of functionally graded auxetic-porous structures was presented by Rad [10] considering torsional interaction and horizontal friction force. The static response of nonuniform bidirectional functionally graded auxetic-porous material circular plates is carried out based on poroelasticity theory. Friction induced a vibration in rotary contact system, and the vibration causes a misalignment problem. Tadokoro et al. [11] described the stabilizing effect arising from parallel misalignment in circular sliding contact. Infinitesimal theory of plane elasticity and Complementary Functions Method was employed to investigate hydrogen-induced stresses in functionally graded axisymmetric spheres, cylinders, and disks by Yildirim [12]. Sondhi et al. [13] used element-based gradation of material properties over the discretized domain to study linear elastic stress analysis of functionally graded rotating disks.

However, the comprehensive understanding and quantifying for the thermoelastic field of a rotating FGM circular disk may be useful in design FGM cutter or a grinding disk. In general, FGM cutter or a grinding disk experiences the thermal load and inertia force due to rotation of the disk. The previous study [14] demonstrated that proper control of some parameters such as temperature distribution, angular speed, and radial thickness improves thermoselastic characteristics in the design of a circular or grinding disk and reduces failure mechanism such as crack and brittle, but most of previous works progressed their studies without considering contact load even though contact load is a crucial parameter in determination of thermoelastic characteristics. The present study focuses on the analysis of thermoelastic characteristics of a rotating $\left(\mathrm{Al}_{2} \mathrm{O}_{3} / \mathrm{Al}\right)$ FGM disk subjected to a contact force. Young's modulus, CTE, and density of the FGM disk are assumed to vary exponentially only in the radial direction due to symmetry with respect to the axis of the disk, whereas Poisson's ratio is assumed to be constant because of insignificant effect on the thermoelastic characteristics of the disk. The research addresses the following: (i) the contact force and homogeneous thickness are crucial factors in the determination of the thermoelastic characteristics of FGM circular models, (ii) the radial direction is more susceptible to the variation of the magnitude of contact force and homogeneous thickness, and (iii) the variation of homogeneous thickness is highly influential to thermoelastic characteristics over the area of $0.2<(r-$ $a) /(b-a)<0.8$ in FGM circular domain.

The process for the mathematical approach is presented in Section 2. A pair of partial differential equations is derived based on Hooke's law in Section 2.1. Due to the complexity of the governing equation, a finite volume method is introduced in Section 2.2 to obtain numerical solutions. In Section 3, numerical solutions are displayed and the elastic characteristics are discussed according to the results obtained. The significances of the results are addressed in the conclusions in Section 4.

\section{Mathematical Modeling}

A rotating FGM circular disk with a concentric circular hole is considered (see Figure 1). The origin of the polar coordinate system $r-\theta$ is assumed to be located at the center of the disk and hole. $A$ and $B$, denoted by the dark and the white colors, represent constituent materials of FGM circular disk, as shown in the figure. The distribution of each material varies continuously along the radial direction only. The radii of the hole and outer surface of the disk are designated by $a$ and $b$. The angular velocity $\omega$ can be determined from the relation $\omega=(2 \pi N / 60), N$ represents the revolutions per minute (rpm). Since the material distributions vary along the radial direction only, the FGM disk can be reduced to an axisymmetric problem. Thus, all properties of the disk can be treated as a function of $r$ only. Young's modulus, coefficient of thermal expansion, and the density of the disk are denoted by $E$, $\alpha$, and $\rho$, respectively, and each property is assumed to vary exponentially as follows (for various and detailed distribution profiles, see [15]):

$$
\begin{aligned}
& E=E_{0} e^{\beta r}, \\
& \alpha=\alpha_{0} e^{\xi r}, \\
& \rho=\rho_{0} e^{\mu r} .
\end{aligned}
$$

By the assumption that the disk is composed of $100 \%$ material $A$ at the surface of the hole $(r=a)$ and $100 \%$ material $B$ at the outer surface $(r=b)$, the constants in equations (1a)-(1c) can be determined as follows:

$$
\begin{aligned}
& E_{0}=E_{A} e^{-\beta a}, \\
& \alpha_{0}=\alpha_{A} e^{-\xi a},
\end{aligned}
$$




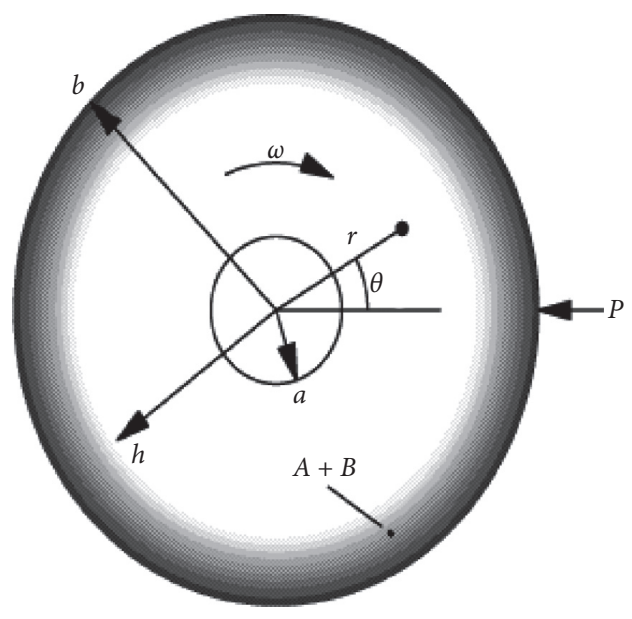

- $A$

$\square B$

(a)

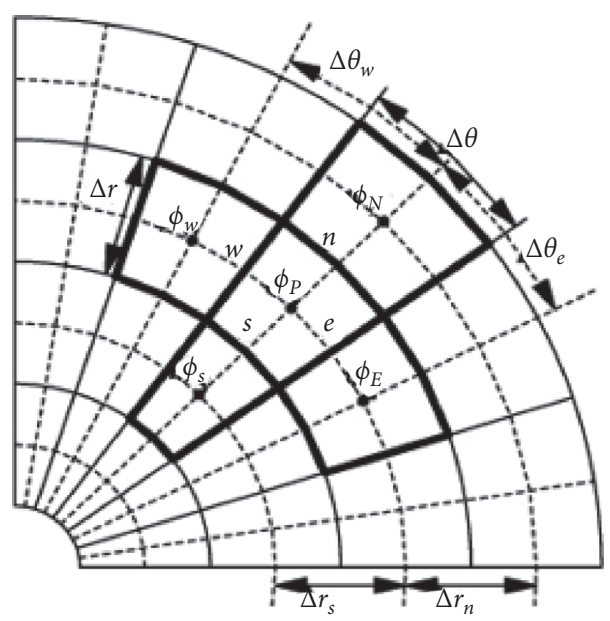

(b)

Figure 1: (a) Schematic diagram of functionally graded circular disk models. (b) Notations of finite control volumes.

$$
\begin{aligned}
& \rho_{0}=\rho_{A} e^{-\mu a}, \\
& \beta=\frac{1}{a-b} \ln \left(\frac{E_{A}}{E_{B}}\right), \\
& \xi=\frac{1}{a-b} \ln \left(\frac{\alpha_{A}}{\alpha_{B}}\right), \\
& \mu=\frac{1}{a-b} \ln \left(\frac{\rho_{A}}{\rho_{B}}\right) .
\end{aligned}
$$

The subscripts $A$ and $B$ represent the properties of the constituent materials $A$ and $B$, respectively, but the nonsubscripted variables are used to denote the properties of FGM composed of materials $A$ and $B$.

2.1. Thermoelastic Formulation. In the polar coordinate, the relations between the strain components and deformation components are as follows:

$$
\begin{aligned}
\varepsilon_{r} & =\frac{\partial u}{\partial r}, \\
\varepsilon_{\theta} & =\frac{1}{r} \frac{\partial v}{\partial \theta}+\frac{u}{r}, \\
\varepsilon_{z} & =0, \\
\gamma_{r \theta} & =\frac{1}{r} \frac{\partial u}{\partial \theta}+\frac{\partial v}{\partial r}-\frac{v}{r}, \\
\gamma_{z \theta} & =0, \\
\gamma_{r z} & =0 .
\end{aligned}
$$

The plane stress exposed with thermal expansion, by Hooke's law, produces the strain-stress relations undergoing thermal expansion ([16], p8):

$$
\begin{aligned}
\varepsilon_{r} & =\frac{1}{E}\left[\sigma_{r}-v \sigma_{\theta}\right]+\alpha T, \\
\varepsilon_{\theta} & =\frac{1}{E}\left[\sigma_{\theta}-v \sigma_{r}\right]+\alpha T, \\
\tau_{r \theta} & =\frac{E}{2(1+\nu)} \gamma_{r \theta}, \\
\tau_{\theta z} & =0, \\
\tau_{r z} & =0 .
\end{aligned}
$$

$T(r)$ is the function of temperature variation with radius variable $r$. The temperature function can be expressed as

$$
T(r)=c_{1} \ln r+c_{2}
$$

under the assumption that the circular disk is subjected by a loading of symmetric temperature to the radial direction only [17]. $c_{1}$ and $c_{2}$ will be determined based on the boundary conditions. The equilibrium equations in polar coordinates are

$$
\begin{array}{r}
\frac{\partial \sigma_{r}}{\partial r}+\frac{1}{r} \frac{\partial \tau_{r \theta}}{\partial \theta}+\frac{\sigma_{r}-\sigma_{\theta}}{r}+\rho \omega^{2} r=0 \\
\frac{\partial \sigma_{\theta}}{\partial \theta}+r \frac{\partial \tau_{r \theta}}{\partial r}+2 \tau_{r \theta}=0
\end{array}
$$

and the algebra among equations (4), (5), (7a), and (7b) yields the following governing equations: 
TABLE 1: Mechanical and thermal properties used for analyzing thermoelastic characteristics of rotating CM circular disks.

\begin{tabular}{|c|c|c|c|}
\hline Material/property & Elastic modulus $(\mathrm{MPa})$ & Thermal expansion coefficient $\left(10^{-6} /{ }^{\circ} \mathrm{C}\right)$ & Density $\left(\mathrm{g} / \mathrm{cm}^{3}\right)$ \\
\hline Substrate $(\mathrm{Al})$ & 71 & 23.1 & 2.70 \\
\hline Bond coating $\left(\mathrm{Al} / \mathrm{Al}_{2} \mathrm{O}_{3}\right)$ & 164.3 & 13.6 & 1.61 \\
\hline Top $\left(\mathrm{Al}_{2} \mathrm{O}_{3}\right)$ & 380 & 8.0 & 0.96 \\
\hline
\end{tabular}

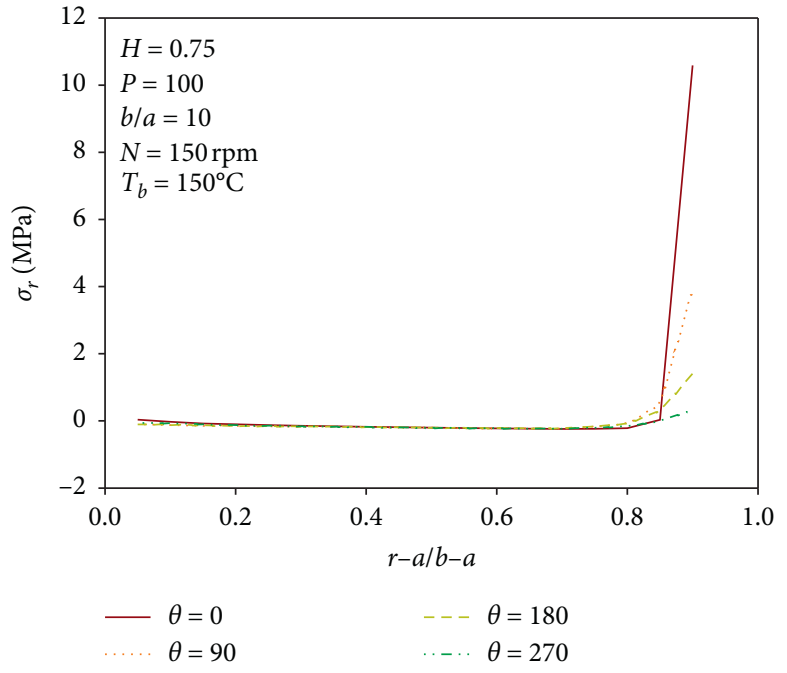

(a)

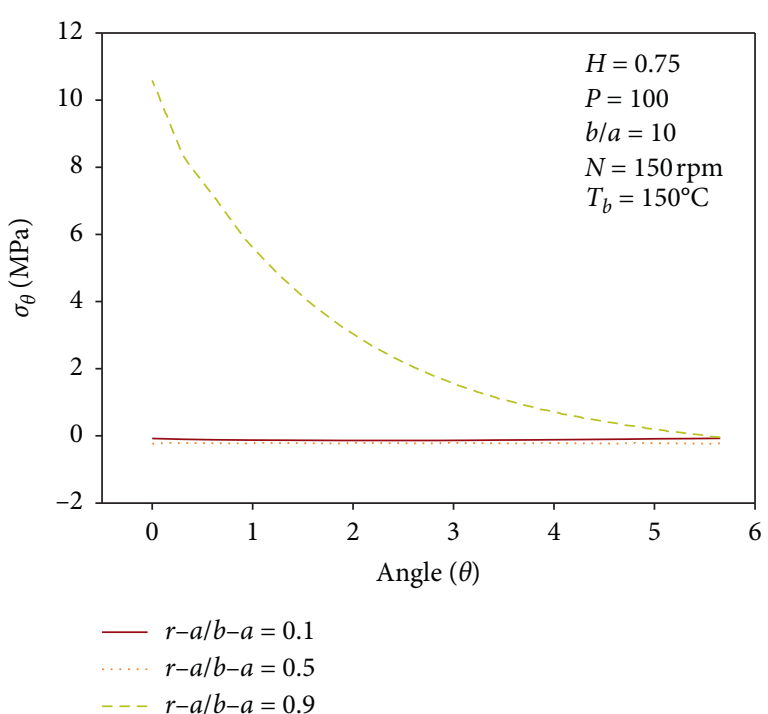

(b)

FIGURE 2: Stress distribution profiles in the (a) radial direction and (b) circumferential direction.

$$
\begin{gathered}
\frac{\partial}{\partial r}\left(r \frac{\partial u}{\partial r}\right)+\frac{1-v}{2 r} \frac{\partial^{2} u}{\partial \theta^{2}}-\frac{3-v}{2 r} \frac{\partial v}{\partial \theta}+\frac{1+v}{2} \frac{\partial^{2} v}{\partial r \partial \theta}-\frac{u}{r} \\
+\frac{1-v^{2}}{E} \rho \omega^{2} r^{2}=(v+1) \alpha r \frac{\mathrm{d} T(r)}{\mathrm{d} r}, \\
\frac{1-v}{2} \frac{\partial}{\partial r}\left(r \frac{\partial v}{\partial r}\right)+\frac{1}{r} \frac{\partial^{2} v}{\partial \theta^{2}}+\frac{3-v}{2 r} \frac{\partial u}{\partial \theta}+\frac{1+v}{2} \frac{\partial^{2} u}{\partial r \partial \theta}-\frac{1-v}{2} \frac{v}{r}=0 .
\end{gathered}
$$

The governing equations (8a) and $(8 b)$ are a pair of partial differential equations in the polar coordinate system expressed with the radial and circumferential displacement distribution profiles under the loading of temperature. The thermoelastic characteristics of a functionally graded rotating circular disk subjected to a contact force are investigated according to the boundary conditions:

$$
\begin{aligned}
\sigma_{r}(a, \theta) & =0, \\
\sigma_{r}(b, \theta-\{0\}) & =0, \\
\sigma_{r}(b, 0) & =P,
\end{aligned}
$$

at a contact point.

The boundary condition implies that the circular model is under the loading of thermal expansion over the entire domain and of radial pressure $P$ at the outer boundary contact point $(b, 0)$.

2.2. Finite Volume Formulation. Due to the complexity of the governing equations, a numerical technique is required, and a finite volume method is applied for approximation. The domain is divided up into control volume and integrates the field equations over each control volume (see Figure $1(\mathrm{~b})$ ). Equations $(8 \mathrm{a})$ and $(8 \mathrm{~b})$ thus are discretized as follows [18]:

$$
\begin{aligned}
& A_{11} u_{i+1, j}+A_{12} u_{i, j+1}+A_{13} u_{i, j}+A_{14} u_{i, j-1}+A_{15} u_{i-1, j} \\
& \quad+B_{11} v_{i, j+1}+B_{12} v_{i, j}+B_{13} v_{i, j-1}+B_{14} v_{i-1, j+1}+B_{15} v_{i-1, j}+B_{16} v_{i-1, j-1}+B_{17} v_{i-2, j+1}+B_{18} v_{i-2, j}+B_{19} v_{i-2, j-1}=f_{i, j} \\
& A_{21} u_{i, j+1}+A_{22} u_{i, j}+A_{23} u_{i, j-1}+A_{24} u_{i-1, j+1}+A_{25} u_{i-1, j}+A_{26} u_{i-1, j-1}+A_{27} u_{i-2, j+1}+A_{28} u_{i-2, j}+A_{29} u_{i-2, j-1} \\
& \quad+B_{21} v_{i+1, j}+B_{22} v_{i, j+1}+B_{23} v_{i, j}+B_{24} v_{i, j-1}+B_{25} v_{i-1, j}=0,
\end{aligned}
$$




$$
\begin{aligned}
& A_{11}=\frac{1-v}{2} \frac{\Delta r}{\Delta \theta} \frac{1}{r_{i, j}}, \\
& A_{12}=\frac{\Delta \theta}{\Delta r} r_{i, j+(1 / 2)} \text {, } \\
& A_{13}=-\frac{\Delta \theta}{\Delta r}\left(r_{i, j+(1 / 2)}+r_{i, j-(1 / 2)}\right)-(1-v) \frac{\Delta r}{\Delta \theta} \frac{1}{r_{i, j}}-\Delta r \Delta \theta \frac{1}{r_{i, j}}, \\
& A_{14}=\frac{\Delta \theta}{\Delta r} r_{i, j-(1 / 2)} \text {, } \\
& A_{15}=\frac{1-v}{2} \frac{\Delta r}{\Delta \theta} \frac{1}{r_{i, j}}, \\
& B_{11}=\frac{3}{8}(1+v) \text {, } \\
& B_{12}=-\frac{3}{4}(3-v) \Delta r \frac{1}{r_{i, j}} \text {, } \\
& B_{13}=-\frac{7}{16}(1+v) \text {, } \\
& B_{14}=-\frac{1}{2}(1+v) \text {, } \\
& B_{15}=\frac{5}{2}(3-v) \frac{1}{r_{i, j}} \\
& B_{16}=\frac{9}{16}(1+v) \text {, } \\
& B_{17}=\frac{1}{8}(1+v), \\
& B_{18}=-\frac{3}{4}(3-v) \Delta r \frac{1}{r_{i, j}} \text {, } \\
& B_{19}=-\frac{1}{8}(1+v) \text {, } \\
& f_{i, j}=(1+v) \alpha r_{i, j} \Delta \theta\left(T_{i, j+(1 / 2)}-T_{i, j-(1 / 2)}\right)-\Delta r \Delta \theta \rho \omega^{2} \frac{1-v^{2}}{E} r_{i, j}^{2} \text {, } \\
& A_{21}=\frac{3}{8}(1+v) \\
& A_{22}=\frac{3}{4}(3-v) \Delta r \frac{1}{r_{i, j}}, \\
& A_{23}=-\frac{7}{16}(1+v) \\
& A_{24}=-\frac{1}{2}(1+v) \\
& A_{25}=-(3-v) \Delta r \frac{1}{r_{i, j}} \\
& A_{26}=\frac{9}{16}(1+v) \text {, } \\
& A_{27}=\frac{1}{8}(1+v) \\
& A_{28}=(3-v) \Delta r \frac{1}{r_{i, j}}, \\
& A_{29}=-\frac{1}{8}(1+v) \\
& B_{21}=\frac{\Delta r}{\Delta \theta} \frac{1}{r_{i, j}}, \\
& B_{22}=\frac{\Delta \theta}{\Delta r} r_{i, j+(1 / 2)},
\end{aligned}
$$




$$
\begin{aligned}
B_{23} & =-\frac{\Delta \theta}{\Delta r}\left(r_{i, j+(1 / 2)}+r_{i, j-(1 / 2)}\right)-\frac{1-v}{2} \Delta r \Delta \theta \frac{1}{r_{i, j}}-2 \frac{\Delta r}{\Delta \theta} \frac{1}{r_{i, j}} \\
B_{24} & =\frac{\Delta \theta}{\Delta r} r_{i, j-(1 / 2)}, \\
B_{25} & =\frac{\Delta r}{\Delta \theta} \frac{1}{r_{i, j}} .
\end{aligned}
$$

The validation and convergence of the above finite volume method (FVM) were presented in [17], and Mathematica (version 5.0) is used to obtain numerical solutions.

\section{Numerical Results and Discussion}

In this section, the differential equation induced in Section 2.1 and the finite volume formula developed in Section 2.2 are used to evaluate the approximated results of different components of displacement, stress, and strain for an $\left(\mathrm{Al}_{2} \mathrm{O}_{3} / \mathrm{Al}\right) \mathrm{FGM}$ circular disk. The mechanical and thermal properties of these ingredient materials are shown in Table 1.

Stress distribution profiles are presented in Figure 2. As shown in Figure 2(a), the circular disk is undergoing tensile radial stress. All areas of the disk except near outer boundary experience trivial influence in radial stress distribution, whereas the contact point suffers from the largest radial stress. But different phase develops in circumferential stress. At normalized values $(r-a) /(b-a)=0.1$ and 0.5 , the magnitude of circumferential stresses is ignorable in comparison with circumferential stress at $(r-a) /(b-a)=0.9$ (see Figure 2(b)). In addition, the entire area of the disk is under the loading of tensile circumferential stress at $(r-a) /(b-a)=0.9$ and the circumferential stress distribution exhibits exponential decay with the increase of angle. The strain distribution profiles are described in Figure 3. Near area of outer boundary in circular domain presents that the magnitude increases with the increase of angle value in the radial strain distribution (see Figure 3(a)). Positive radial strain distribution appears over the disk, and the largest radial strain value occurring at the contact point generates. However, the circumferential strain distribution shows different pattern (see Figure 3(b)). At $(r-a) /(b-a)=0.9$, the circumferential strain deploys to the positive direction, while negative circumferential strain distributions are obtained at $(r-a) /(b-a)=0.1$ and 0.5 . The changing shape of the circumferential strain is a parabola as the angle increases at $(r-a) /(b-a)=0.1$ and 0.5 , whereas the circumferential strain distribution profile fluctuates at the beginning and logarithmic decay appears after $\theta=2$ at $(r-a) /(b-a)=0.9$. The results inform that (i) contact force is highly influential to near area of contact point in radial direction, (ii) the circumference stress is more sensitive to contact force than the radial stress distribution, and (iii) various phenomena appear in circumferential strain distribution depending on the value of $(r-a) /(b-a)$.

Figure 4 presents the influences of contact force on the stress distribution by choosing the representative angles $\theta=$ 0 and 180 and the normalized radius $(r-a) /(b-a)=0.1$ and 0.9. The radial stress distribution is susceptible to the change of contact force, especially near the area of contact point (see Figures 4(a) and 4(b)). Entire domain of the circular disk suffers from a tensile stress and the magnitude of the radial stress is getting larger as the contact force increases. As shown in Figure 4(c), the area near the inner boundary of the disk sits loose to the variation of contact force, but the area near the outer boundary reacts sensitively to the change of contact force, and noticeable impact appears when $\theta=0$ (see Figure 4(d)). By the increase of contact force, the radial and circumferential stresses exhibit exponential growth near area of contact point, even though minor effects appear over entire circular domain.

The effects of contact force on the strain distributions are described in Figure 5. The reactions of the radial strain distribution to the change of contact force are similar to those of radial stress distribution (see Figures 5(a) and 5(b)). Nearby area of the outer boundary is particularly susceptible to the increase of contact force and the magnitude of the radial strain grows with the increment of contact force. Trivial reaction appears in the circumferential strain distribution to the variation of contact force for $(r-a) /(b-a)=0.1$ (see Figure 5(c)), whereas nearby part of the outer boundary is sensitive to the change of contact force (see Figure 5(d)). Pronounced alterations in the circumferential strain distribution appear on area around $\theta=0$. The numerical solutions imply that (i) the magnitude of contact force is a critical factor to determine the thermoelastic characteristics of circular FGM models and (ii) the radial direction is more susceptible to the variation of the magnitude of contact force.

The influences of homogeneous thickness on thermoelastic characteristics, denoted by $\mathrm{H}$, are explained through Figures 6-8. $\mathrm{H}=1$ implies that the circular disk is composed of homogeneous material. The representative values $\mathrm{H}=0.25,0.5$, and 0.75 are applied. Figure 6 expresses the effects of $\mathrm{H}$ on the displacement distributions.

The area between normalized values $(r-a) /(b-a)=$ 0.2 and $(r-a) /(b-a)=0.85$ reveals noticeable reactions to homogeneous thickness variation. The magnitude of the radial displacement increases with the growth of homogeneous part (see Figures 6(a) and 6(b)), but nearby area of the outer boundary displays that the magnitude to the positive direction decreases as the value of $\mathrm{H}$ increases. The results imply that the growth of functionally graded area in the circular disk yields more stability in the radial displacement distribution profiles.

As shown in Figures 6(c) and 6(d)), minor influence appears in the circumferential displacement except nearby area of contact force. The circumferential displacement 


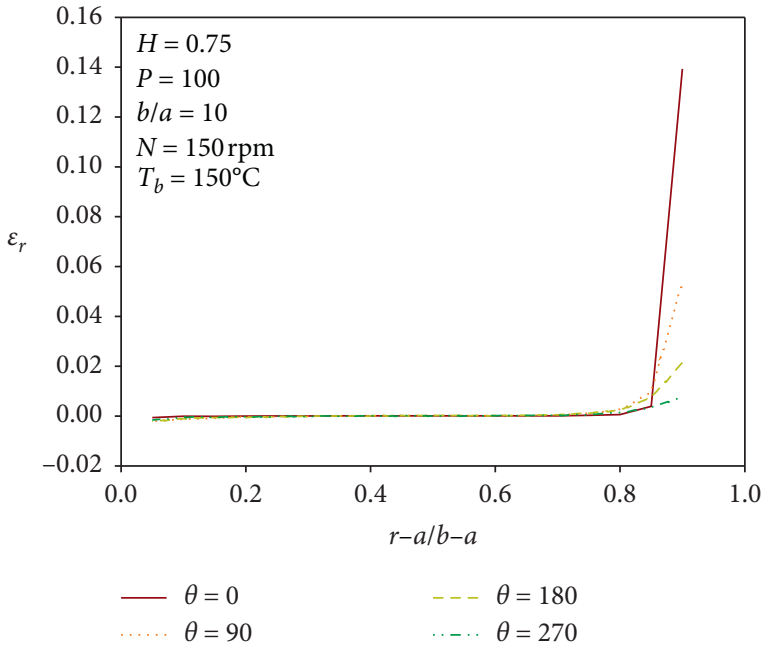

(a)

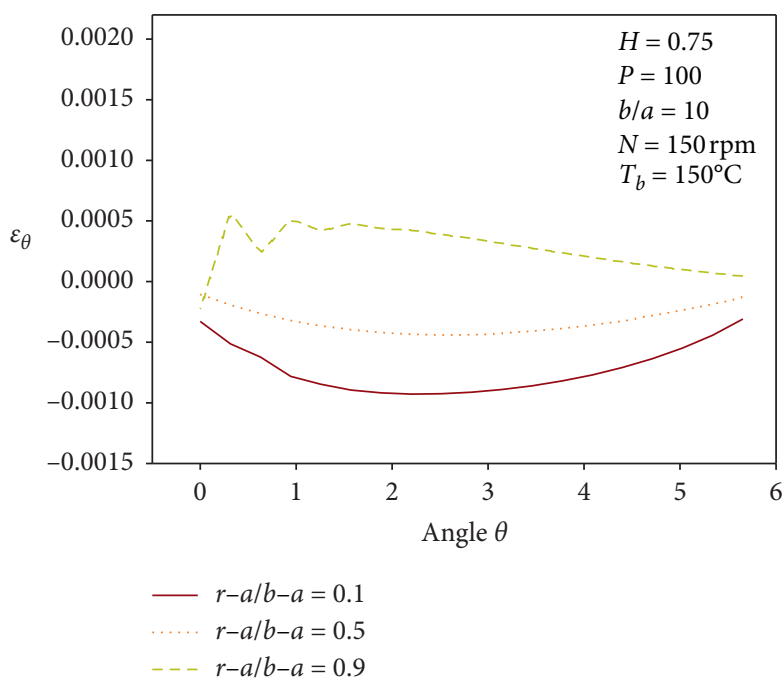

(b)

FIgURE 3: Strain distribution profiles in the (a) radial direction and (b) circumferential direction.

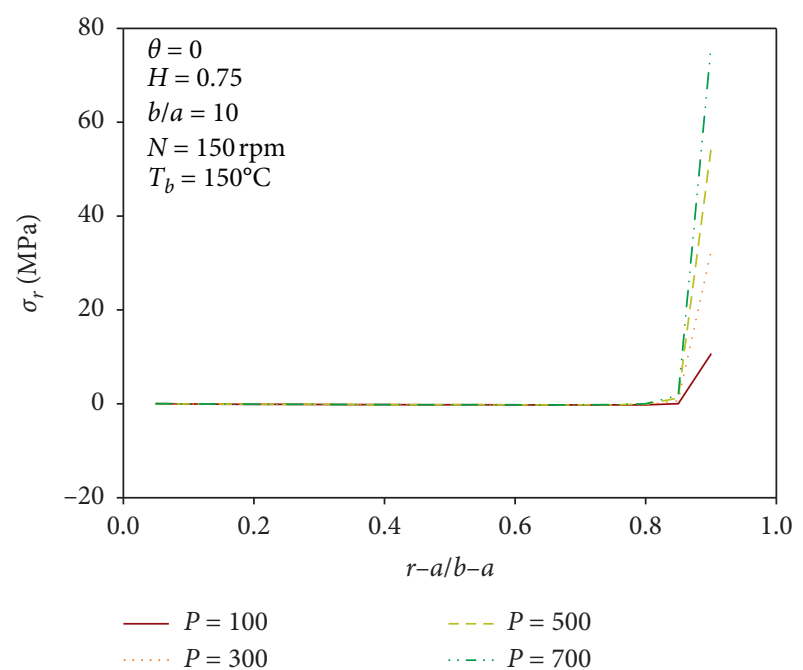

(a)

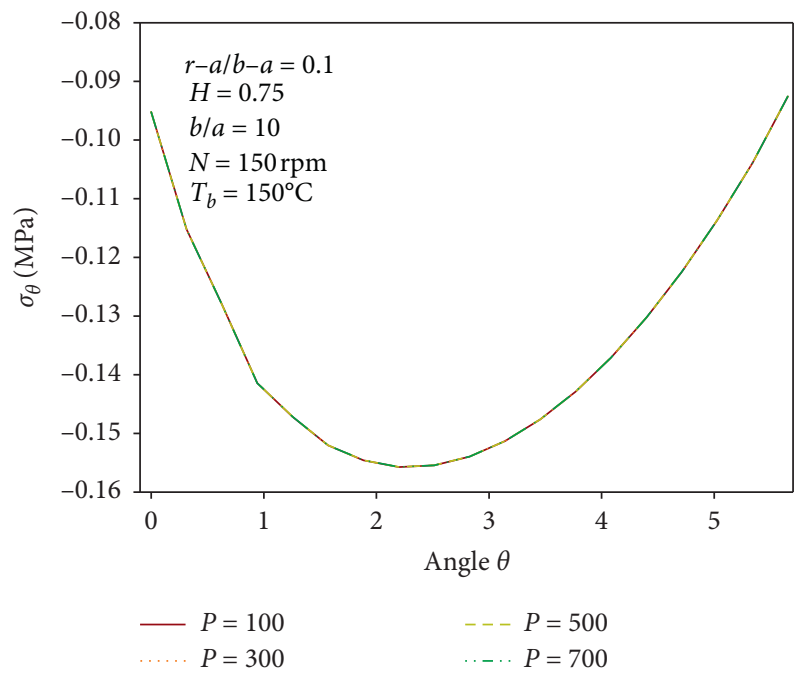

(c)

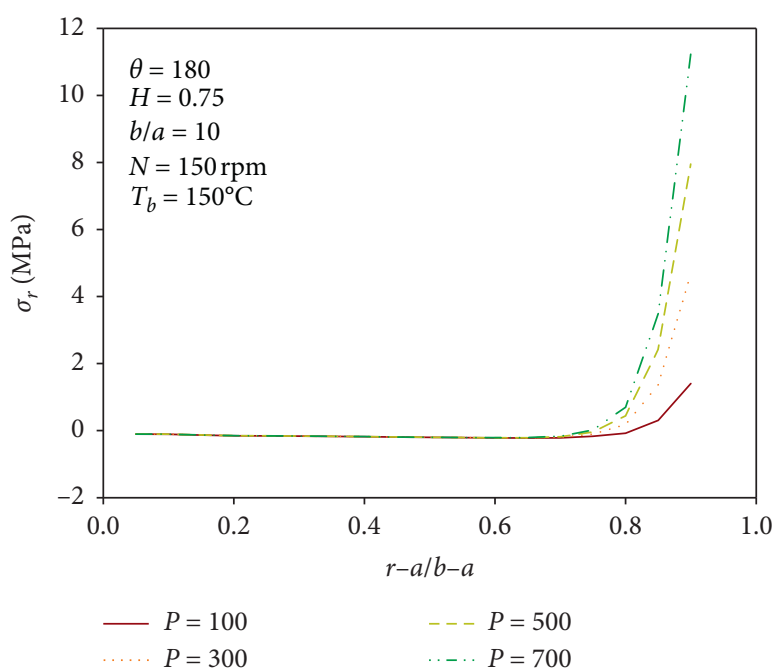

(b)

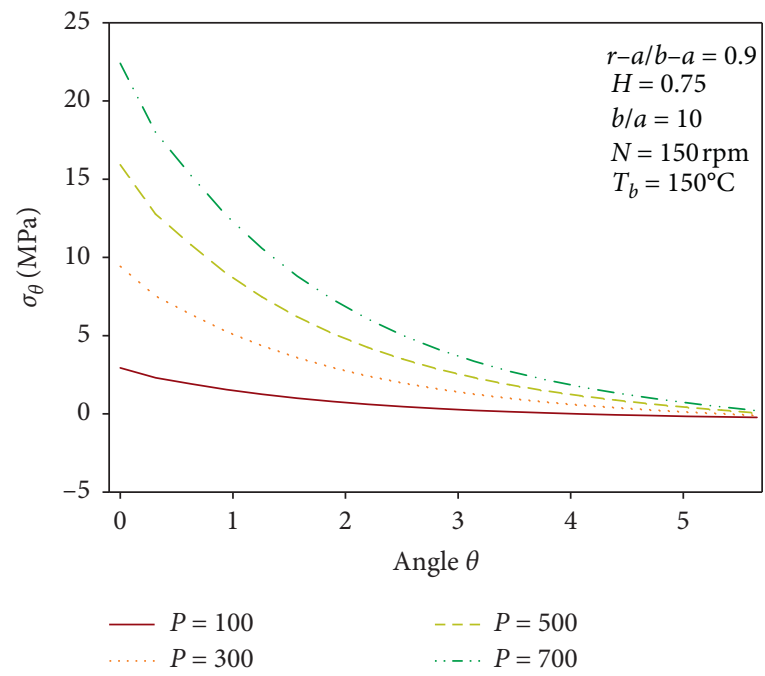

(d)

FIgURE 4: Effects of contact force on the stress in the radial direction (a) at $\theta=0$ and (b) at $\theta=180$ and in the circumferential direction (c) at $(r-a) /(b-a)=0.1$ and $(\mathrm{d})$ at $(r-a) /(b-a)=0.9$. 


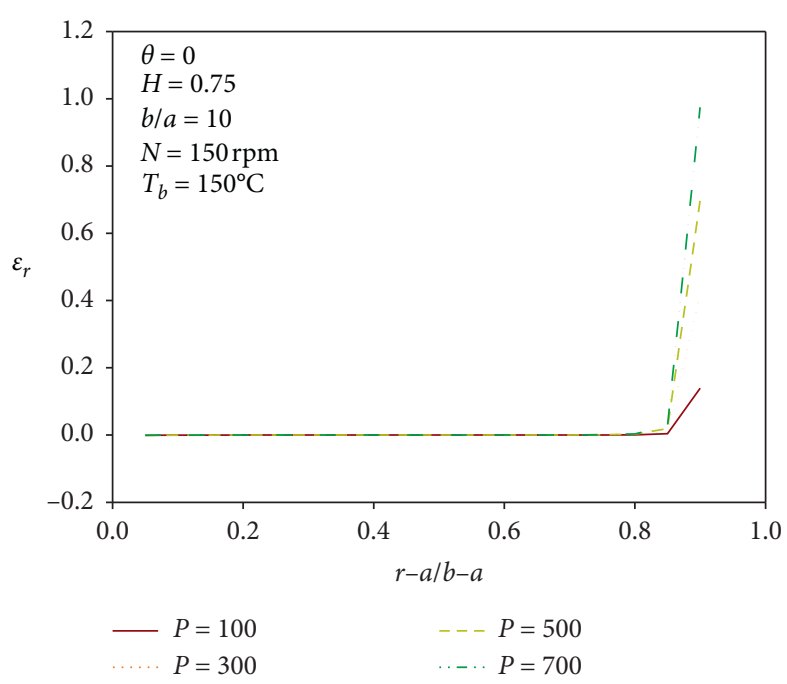

(a)

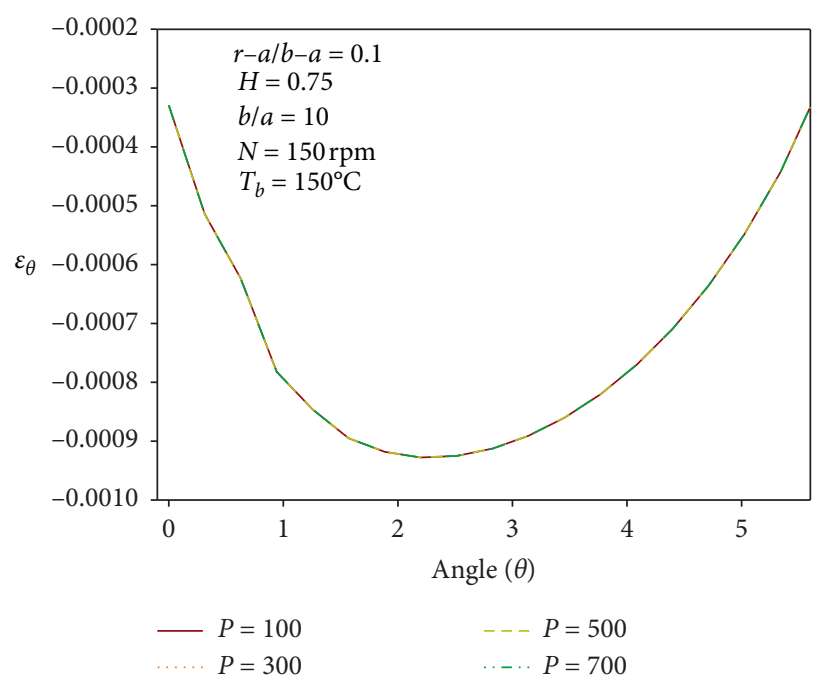

(c)

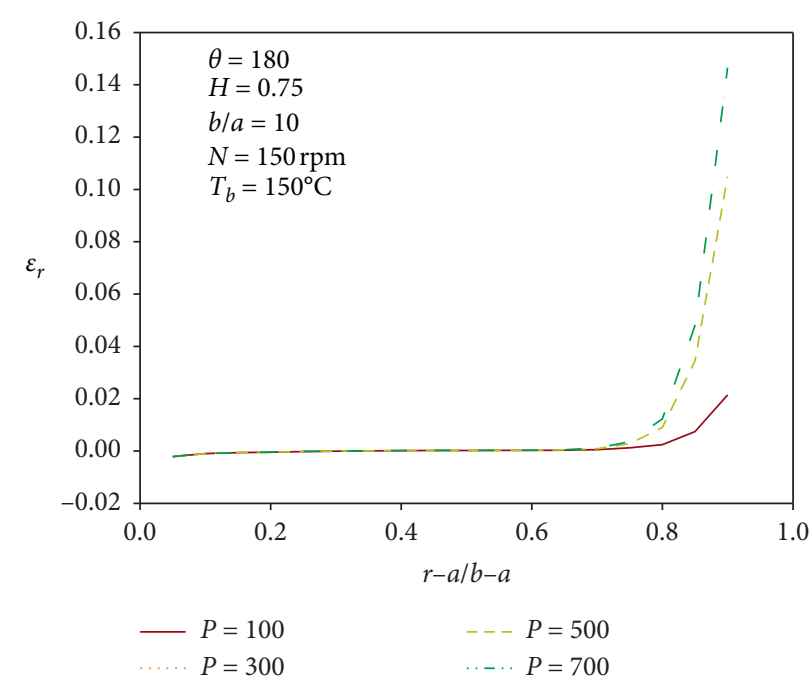

(b)

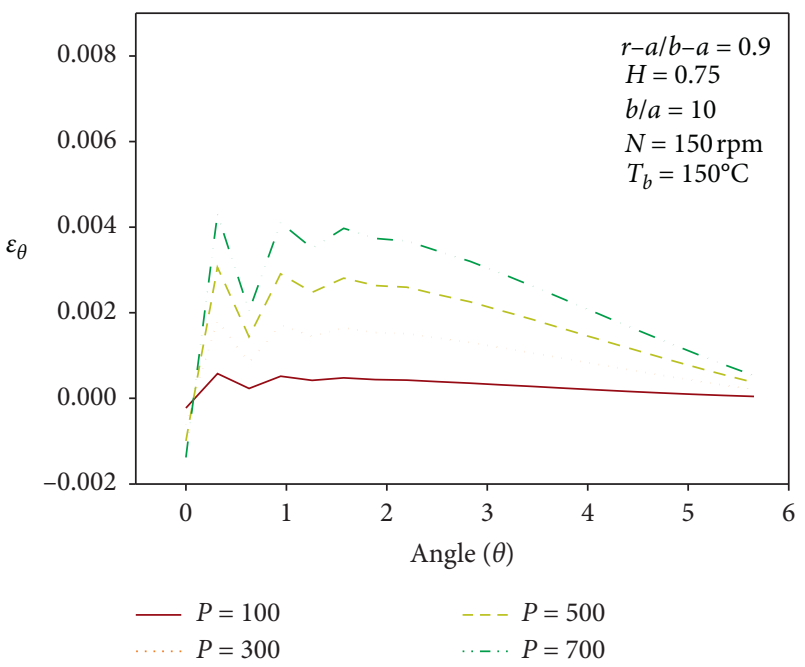

(d)

FIGURE 5: Effects of contact force on the strain in the radial direction (a) at $\theta=0$ and (b) at $\theta=180$ and in the circumferential direction (c) at $(r-a) /(b-a)=0.1$ and $(\mathrm{d})$ at $(r-a) /(b-a)=0.9$.

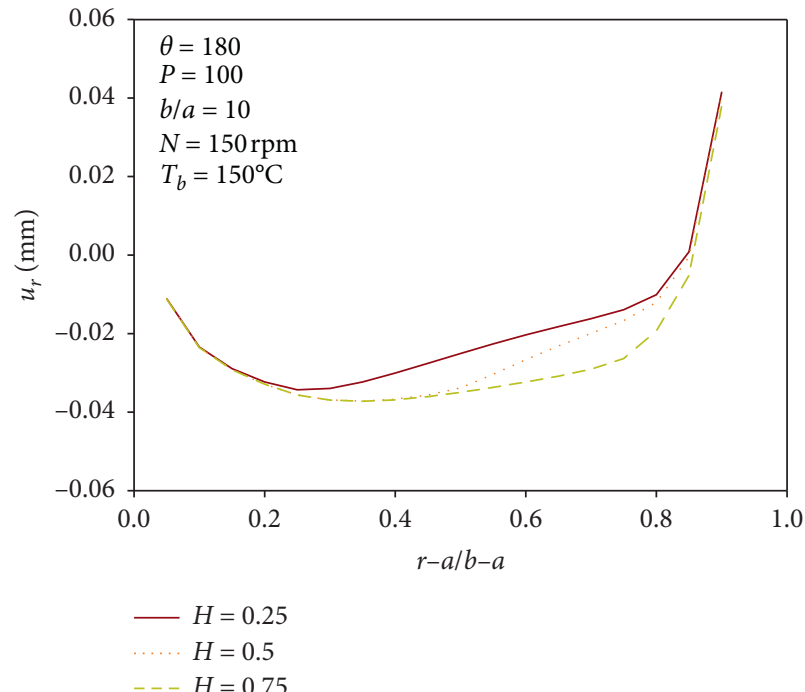

(a)

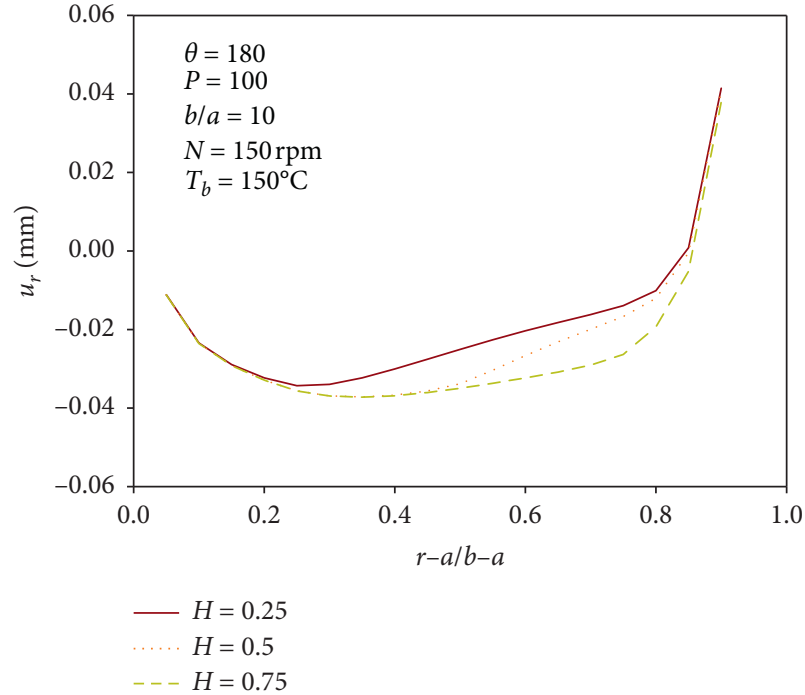

(b)

FIgURe 6: Continued. 


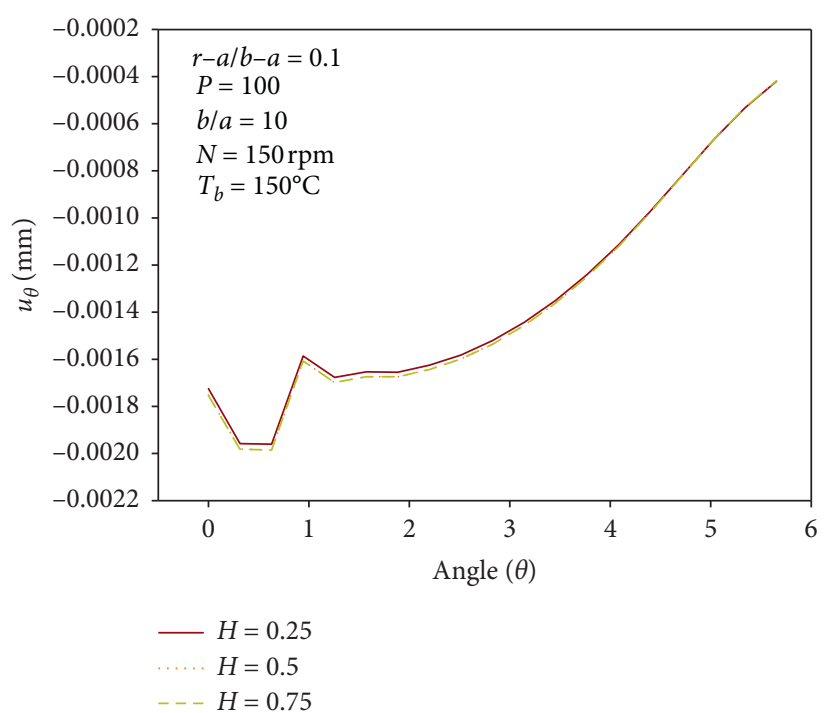

(c)

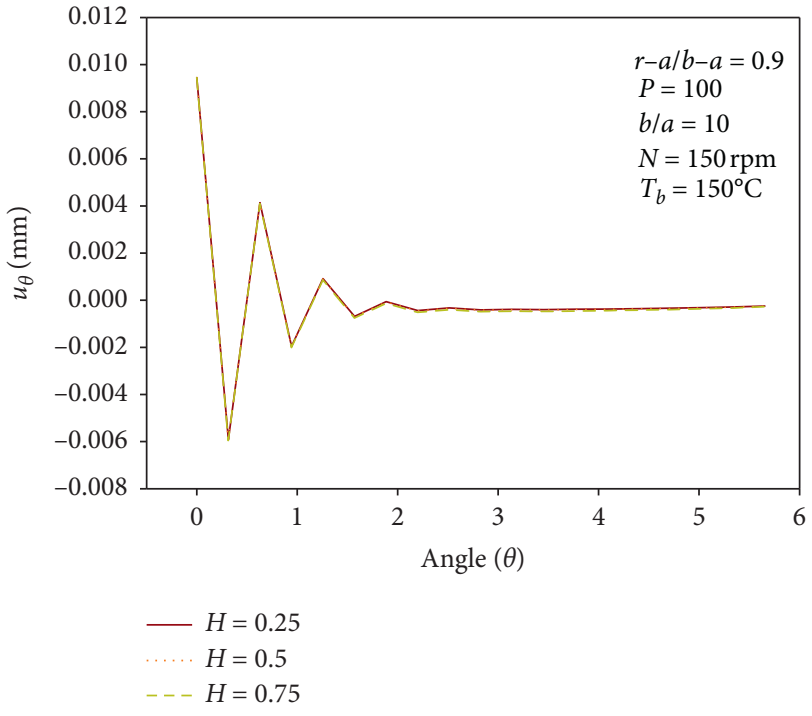

(d)

Figure 6: Effects of homogeneous thickness on the displacement in the radial direction (a) at $\theta=0$ and (b) at $\theta=180$ and in the circumferential direction (c) at $(r-a) /(b-a)=0.1$ and $(\mathrm{d})$ at $(r-a) /(b-a)=0.9$.

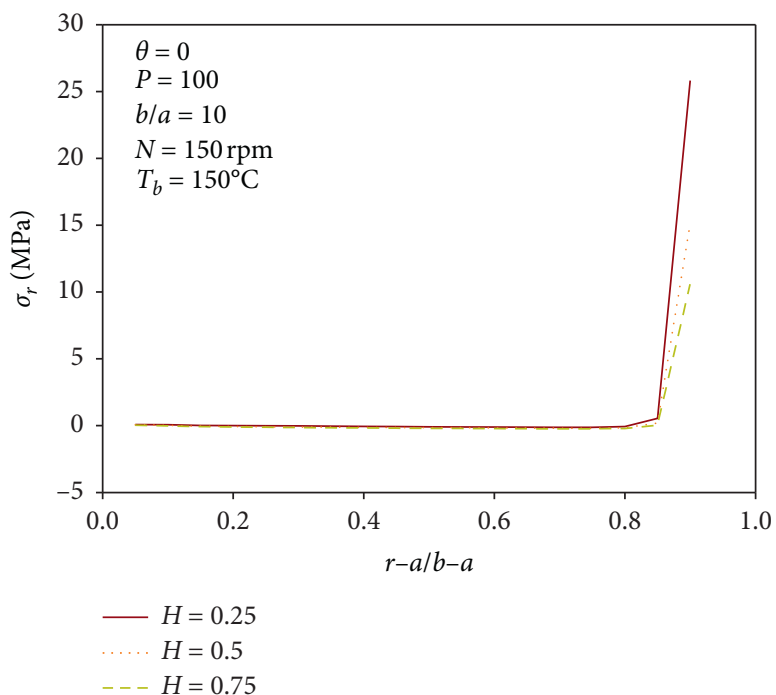

(a)

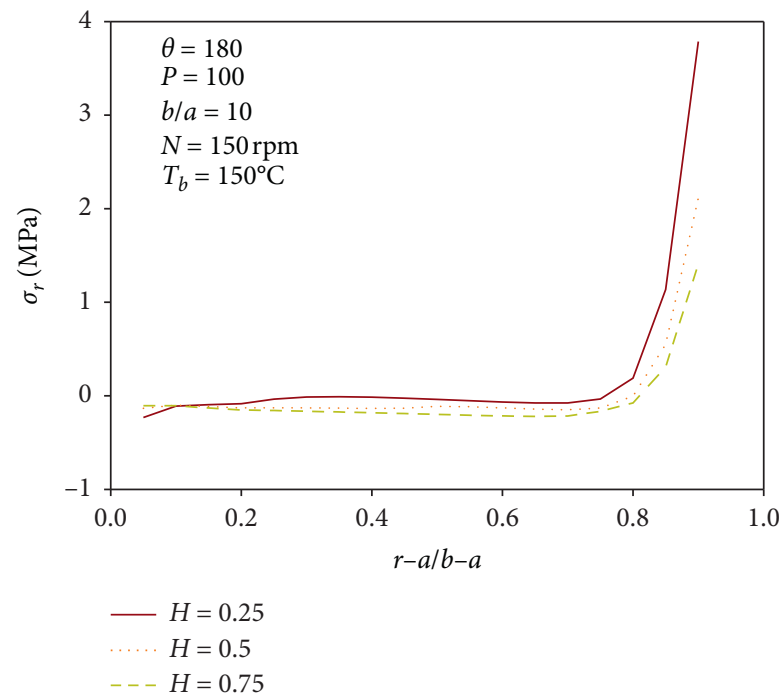

(b)

Figure 7: Continued. 


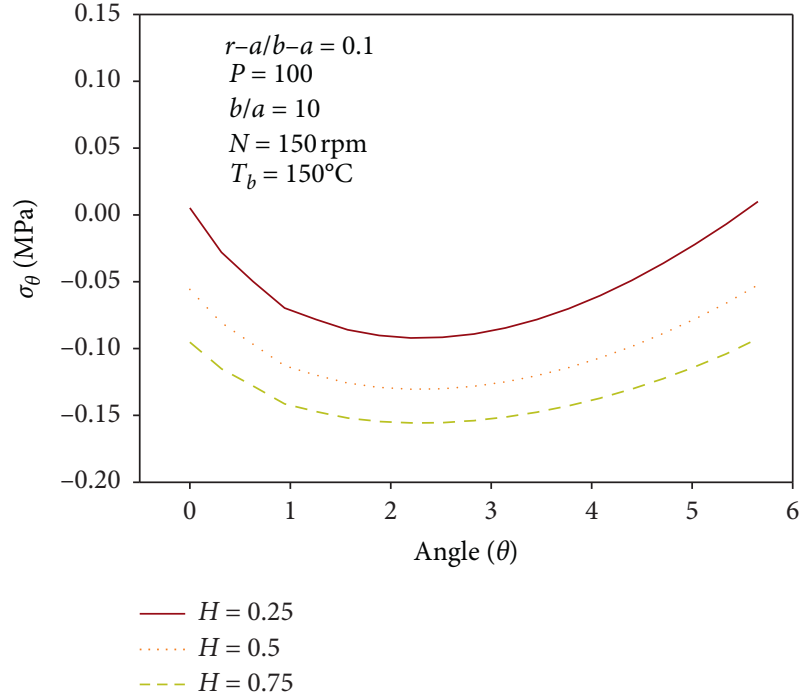

(c)

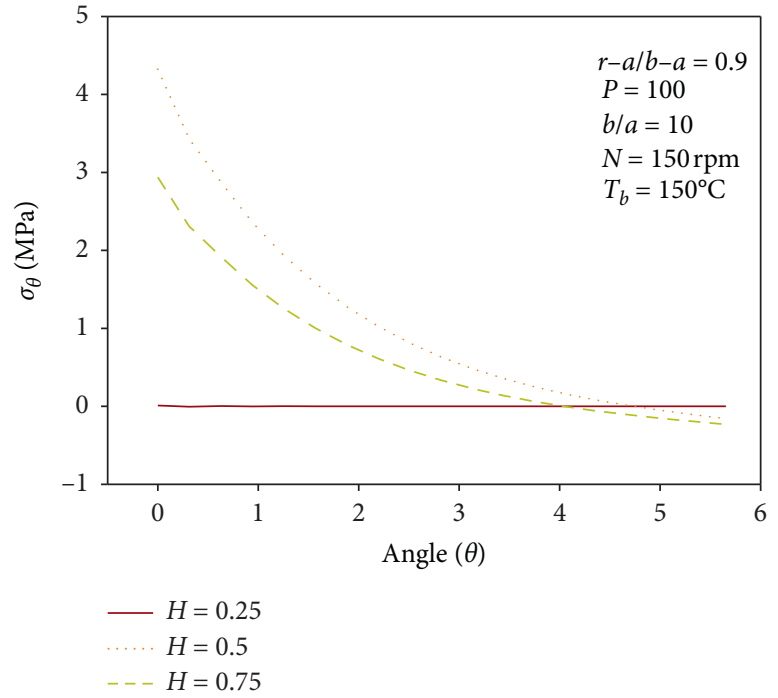

(d)

Figure 7: Effects of homogeneous thickness on the stress in the radial direction (a) at $\theta=0$ and (b) at $\theta=180$ and in the circumferential direction $(\mathrm{c})$ at $(r-a) /(b-a)=0.1$ and $(\mathrm{d})$ at $(r-a) /(b-a)=0.9$.

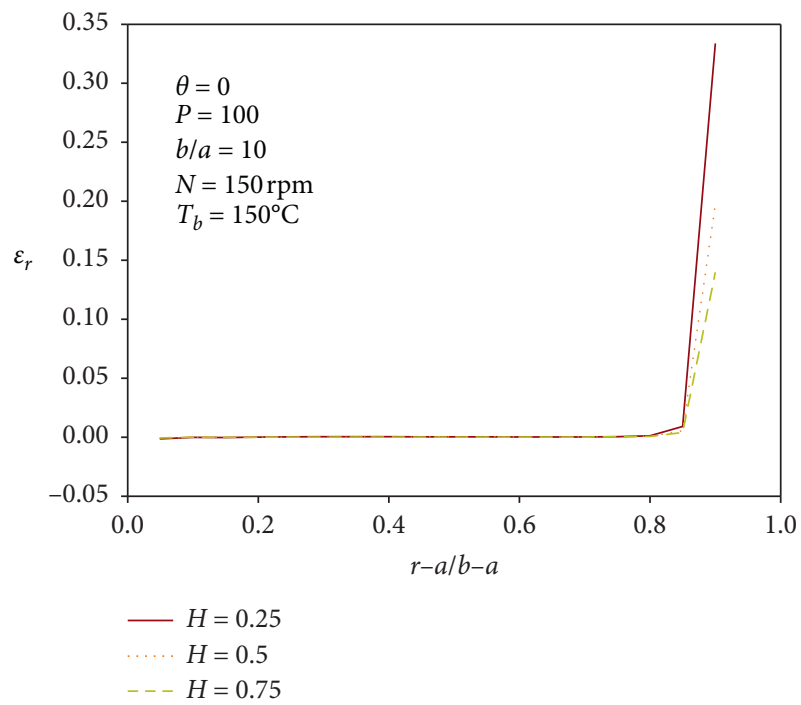

(a)

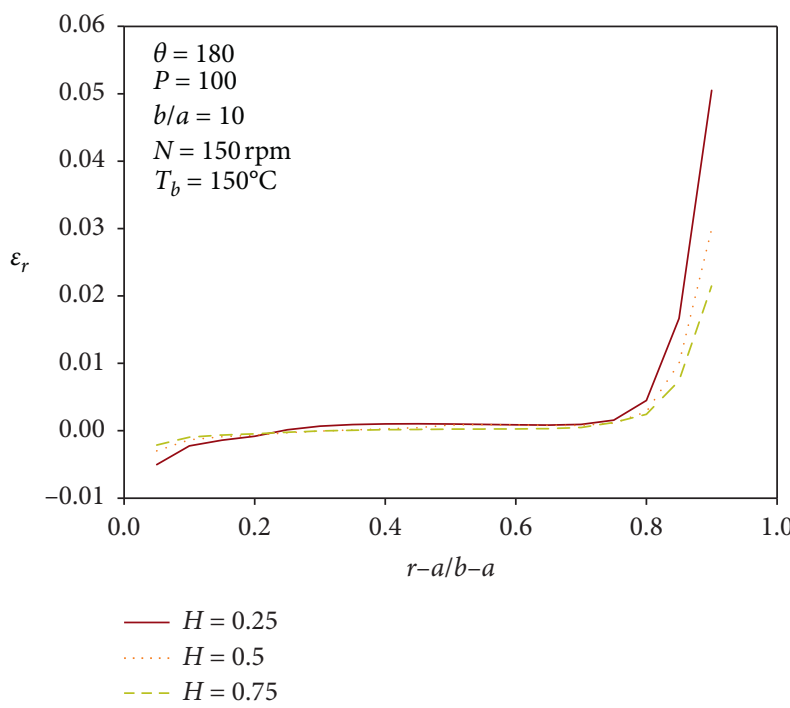

(b)

Figure 8: Continued. 


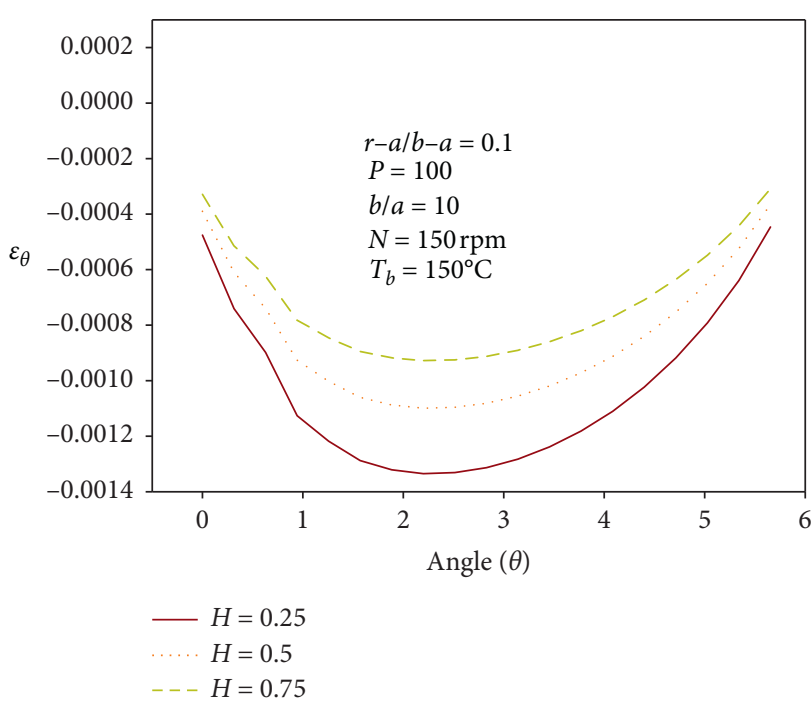

(c)

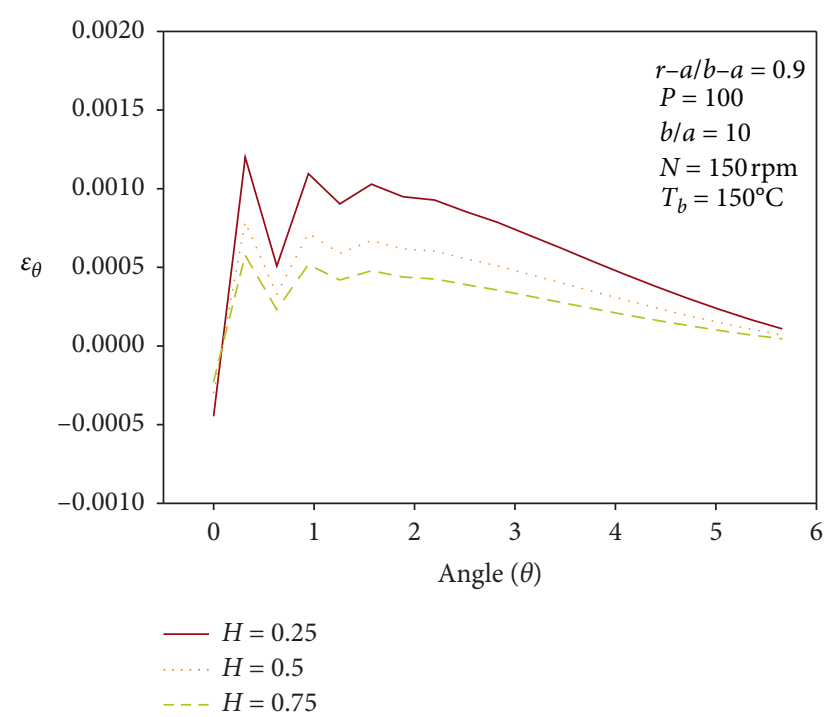

(d)

FiguRE 8: Effects of homogeneous thickness on the strain in the radial direction (a) at $\theta=0$ and (b) at $\theta=180$ and in the circumferential direction $(\mathrm{c})$ at $(r-a) /(b-a)=0.1$ and $(\mathrm{d})$ at $(r-a) /(b-a)=0.9$.

increases with growth of the value of $\mathrm{H}$ over the area around contact force.

Figure 7 presents the effects of $\mathrm{H}$ on the stress distributions. The radial distribution exhibits smaller tensile stress over the nearby area of outer boundary with increase of the value of $\mathrm{H}$ when $\theta=0$ (see Figure $7(\mathrm{a})$ ). But the circular disk undergoes larger compressive radial stress over area between normalized values $(r-a) /(b-a)=0.1 \quad$ and $(r-a) /(b-a)=0.8$ when $\theta=180$ (see Figure $7(b))$. According to the radial stress distributions, the circular disk with larger functionally graded area may not guarantee higher stability over nearby area of outer boundary. Different phase develops in circumferential stress distributions. Over nearby area of the inner boundary, entire domain of the disk experiences compressive circumferential stress for the values of $\mathrm{H}=0.5$ and 0.75 , whereas some part of the disk with $\mathrm{H}=0.25$ undergoes tensile circumferential stress (see Figure $7(\mathrm{c})$ ). Over nearby area of the outer boundary of the disk, the circumferential stress with $\mathrm{H}=0.75$ is trivial in comparison with the value of $\mathrm{H}=0.25$ and 0.5 (see Figure $7(\mathrm{~d})$ ). The magnitude of the circumferential stress is higher near contact point as the homogeneous thickness decreases. Based on the circumferential stress distributions, greater functionally graded area affords higher stability in the circular disk. The influences of homogeneous thickness $\mathrm{H}$ on the strain distributions are described in Figure 8. Nearby area of the outer boundary of the disk is sensitive to the change of homogeneous thickness in the radial strain distributions. The magnitude of strain decreases with the growth of the value of $\mathrm{H}$ (see Figures 8(a) and 8(b)), which signifies that the circular disk with larger homogeneous area is more stable over nearby area of the outer boundary. Similar movements occur in the circumferential strain. As shown in Figures 8(c) and 8(d), the magnitude of the circumferential strain is getting larger according to the decline of the value of $\mathrm{H}$. The outcomes describe that the circular disk stability with greater functionally graded area is greater in the circumferential strain distribution. The numerical approaches make point of the following: (i) the variation of homogeneous thickness is highly influential to thermoelastic characteristics over the area of $0.2<(r-a) /(b-a)<0.8$ and (ii) the radial direction is more susceptible to the change of homogeneous thickness.

\section{Conclusions}

A rotating FGM circular disk with a concentric circular hole is taken into account to investigate the thermoelastic characteristics. The circular disk is under the loading of a contact force. The effects of the variation of contact force and homogeneous thickness on the components of displacement, stress, and strain have been described. Over nearby area of the contact point, the thermoselastic behavior reacts sensitively to the change of contact force and exhibits exponential growth in the magnitude, whereas the influence of contact force over the other area is greater in the circumferential stress distributions. The outcomes explain that the outer boundary area is exposed to become brittle with the growth of a contact force. In the radial displacement distribution, the growth of functionally graded area grants more stability in the circular disk, while, according to the radial stress distributions, the circular disk with larger functionally graded area may not guarantee higher stability over nearby area of outer boundary. Based on the circumferential distribution profiles, the circular disk with greater functionally area evinces higher stability. Overall, the results demonstrate that contact force and homogeneous thickness are crucial parameters to determine the thermoelastic characteristics of FGM circular disks, and the movements of an FGM circular disk can be controlled by 
controlling of these parameters. Therefore, a FGM circular cutter or grinding disk suffering from the loading of a contact force can be designed applying the finite volume analysis used in this investigation to promote proper and reliable thermoelastic characteristics in service.

\section{Nomenclature}

$u$ : Radial displacement component ( $\mathrm{mm}$ )

$v$ : Circumferential displacement component ( $\mathrm{mm})$

$\varepsilon_{r}:$ Radial strain

$\varepsilon_{\theta}$ : Circumferential strain

$\gamma$ : Shearing strain

$\sigma_{r}:$ Radial stress $(\mathrm{MPa})$

$\sigma_{\theta}:$ Circumferential stress $(\mathrm{MPa})$

$\tau$ : $\quad$ Shearing stress

$v$ : Poisson's ratio

$\omega$ : Angular velocity

$N$ : Revolutions per minute (rpm)

$E$ : Young's modulus ( $\mathrm{MPa})$

$\alpha$ : Coefficient of thermal expansion $\left(10^{-6} /{ }^{\circ} \mathrm{C}\right)$

$\rho$ : Density of the disk $\left(\mathrm{g} / \mathrm{cm}^{3}\right)$.

\section{Data Availability}

The data used to support the findings of this study are available from the corresponding author upon request.

\section{Conflicts of Interest}

The author declares that there are no conflicts of interest.

\section{Acknowledgments}

This research was supported by Basic Science Research Program through the National Research Foundation of Korea (NRF) funded by the Ministry of Education (NRF2018R1D1A1B07043714).

\section{References}

[1] J. B. Holt, M. Koizumi, T. Hirai, and Z. A. Munir, Ceramic Transactions, Functionally Gradient Materials, The American Ceramic Society, p. 34, Westerville, Ohio, USA, 1993.

[2] K. BnvsGaneshGupta, M. M. Hiremath, A. Fulmali, R. K. Prusty, and B. C. Ray, "Multimaterial laminated composites: an assessment of effect of stacking sequence on flexural response," Material Today: Proceedings, 2020.

[3] N. Zimmermann and P. H. Wang, "A review of failure modes and fracture analysis of aircraft composite materials," Engineering Failure Analysis, vol. 115, Article ID 104692, 2020.

[4] Y. Obata and N. Noda, "Steady thermal stresses in a hollow circular cylinder and a hollow sphere of a functionally gradient material," Journal of Thermal Stresses, vol. 17, no. 3, pp. 471-487, 1994.

[5] K. M. Liew, S. Kitipornchai, X. Z. Zhang, and C. W. Lim, "Analysis of the thermal stress behaviour of functionally graded hollow circular cylinders," International Journal of Solids and Structures, vol. 40, no. 10, pp. 2355-2380, 2003.

[6] A. M. Afsar, M. Anisuzzaman, and J. I. Song, "Inverse problem of material distribution for desired fracture characteristics in a thick-walled functionally graded material cylinder with two diametrically-opposed edge cracks," Engineering Fracture Mechanics, vol. 76, no. 7, pp. 845-855, 2009.

[7] Y. Kovalyshen, "Analytical model of oscillatory disc cutting," International Journal of Rock Mechanics and Mining Sciences, vol. 77, pp. 378-383, 2015.

[8] Y. Huang and X.-F. Li, "Effect of radial reaction force on the bending of circular plates resting on a ring support," International Journal of Mechanical Sciences, vol. 119, pp. 197-207, 2016.

[9] H.-L. Dai, T. Dai, and X. Yan, "Thermoelastic analysis for rotating circular HSLA steel plates with variable thickness," Applied Mathematics and Computation, vol. 268, pp. 10951109, 2015.

[10] A. B. Rad, "Static analysis of non-uniform 2D functionally graded auxetic-porous circular plates interacting with the gradient elastic foundations involving friction force," Aerospace Science and Technology, vol. 76, pp. 315-339, 2018.

[11] C. Tadokoro, T. Nagamine, and K. Nakano, "Stabilizing effect arising from parallel misalignment in circular sliding contact," Tribology International, vol. 120, pp. 16-22, 2018.

[12] S. Yildirim, "Hydrogen elasticity solution of functionallygraded spheres, cylinders and disks," International Journal of Hydrogen Energy, vol. 45, no. 41, pp. 22094-22101, 2020.

[13] L. Sondhi, A. Kumar Thawait, S. Sanyal, and S. Bhowmick, "Stress and deformation analysis of variable thickness clamped rotating disk of functionally graded orthotropic material," Materials Today: Proceedings, vol. 18, pp. 4431-4440, 2019.

[14] J. Go, A. M. Afsar, and J. I. Song, "Analysis of thermoelastic characteristics of a rotating FGM circular disk by finite element method," Advanced Composite Materials, vol. 19, no. 2, pp. 197-213, 2010.

[15] A. M. Afsar and J. Go, "Finite element analysis of thermoelastic field in a rotating FGM circular disk," Applied Mathematical Modelling, vol. 34, no. 11, pp. 3309-3320, 2010.

[16] S. P. Timoshenko and J. N. Goodier, Theory of Elasticity, McGraw-Hill Book Company, New York, NY, USA, 3rd edition, 2010.

[17] M. Tadi, "Finite volume method for 2D elastic wave propagation," Bulletin of the Seismological Society of America, vol. 94, no. 4, pp. 1500-1509, 2004.

[18] J. Go, "Thermo-elastic characteristics of homogeneous circular disk models subjecting to a contact force," Contemporary Engineering Sciences, vol. 12, no. 1, pp. 51-64, 2019. 